\title{
Advanced uses of Stress Intensity Factor Tables in PTS Calculations of Nuclear Power Plants
}

\author{
Tamás Pálfi' ${ }^{1, *}$ \\ VEIKI Energia+ Ltd, 47 Murányi utca, Budapest, 1078 Hungary
}

\begin{abstract}
This paper introduces an approximation method developed for calculating stress intensity factor for pressure vessels in nuclear power plants. The method is based on weight functions, which has high accuracy among the methods based on crack-free stress calculations. Besides it has the advantage over the weight functions method, it corresponds to the approximation methods can be found in nuclear codes like ASME BPVC [1]. Numerical test calculations are performed to experimentally show the differences of several possible approximation methods. The results show the weighted least squares method using stress intensity factor weight functions, do have the above-mentioned advantages.
\end{abstract}

Keywords: fracture mechanics; stress intensity factors; weight functions; nuclear power plants;

\section{Introduction}

Stress intensity factor calculations in applied fracture mechanical analyses for nuclear power plants are mostly based on the polynomial regression of the crack-free stress field, postulating planar semi-elliptical surface cracks. The importance of such calculations is shown by the fact, that the scope of fracture mechanics analyses for power plant safety reports and aging management generally covers:

" Reactor pressure vessel brittle fracture, crack propagation and PTS (Pressurized Thermal Shock) analysis;

" Brittle fracture and crack propagation analyses of main circulation pipes;

"Brittle fracture and crack propagation analyses of the pressurizer vessel;

» Brittle fracture and crack propagation analyses of other primary circuit equipment and pipelines.

\section{Summary of generally used methods}

\subsection{Methods based on polynomial regression}

Nuclear authorities usually expect calculation procedures set out in nuclear codes for the above listed analysis. These procedures are most commonly based on the following scheme (for example: [1] Appendix A-3000):

Polynomial regression of the crack opening stress calculated by the FEM analyses:

$$
\sigma^{F E M}\left(\frac{x}{a}\right) \approx \sigma^{A}\left(\frac{x}{a}\right)=A_{0}+A_{1}\left(\frac{x}{a}\right)+A_{2}\left(\frac{x}{a}\right)^{2}+A_{3}\left(\frac{x}{a}\right)^{3},
$$

where $x$ is the coordinate normal to the vessel's inner surface, a is the crack depth, $\sigma^{F E M}$ is the distribution of the stress component normal to the crack plane, calculated by the Finite Element Model (FEM), $\sigma^{A}$ is the polynomial regression of $\sigma^{F E M}$, with the regression coefficients: $A_{0}, A_{1}, A_{2}$ and $A_{3}$. Determining regression coefficients might be performed for a given $\sigma^{F E M}$ function, although the code does not specify its method.

Stress intensity factor $\left(K_{I}\right)$ is calculated using the determined regression coefficients:

$$
K_{I}^{G}=R_{0}\left[\left(A_{0}+A_{p}\right) G_{0}+A_{1} G_{1}+A_{2} G_{2}+A_{3} G_{3}\right] \text {, }
$$


where $a$ is crack size; $l$ is crack surface length, $A_{p}$ is internal pressure the $G_{0}, G_{1}, G_{2}, G_{3}$ coefficients are determined using the tables in the code, and $R_{0}$ is a coefficient containing the crack shape correction factor and the crack tip plastic zone correction factor.

Main characteristics of the above method:

"The curve regression method is not specified by the code, and the base polynomials of the regression - which are the $\left(\frac{x}{a}\right)^{i}, i=$ $0,1,2,3$ terms in this case - are not numerically optimized. These follows, that significant difference of the result might occur using in two different regressions for the same FEM results.

"It is not suitable for jump discontinuities of the stress function, or for any other characteristics which cannot be approximated sufficiently with polynomial regression. Such anomalies are caused, for example by stresses due to different thermal expansion coefficient of the cladding or residual stresses according to nuclear codes, etc.

"The coefficients might contain the conservatism of this method. This conservatism might not be neglected by changing to another similar method, in a case when this method is adopted in a regulation or a guideline by the authority, or it is a part of the basic nuclear standard of the power plant, or some form of other official regulations.

\subsection{Specific weight functions}

Another way to calculate the stress intensity factor is the method of weight functions, which is with collected coefficients - written in the following form:

$K_{I}^{w}=R_{w} \int_{0}^{a} w(x) \sigma^{V E M}(x) d x$,

where $R_{w}$ is a coefficient calculated from the given crack configuration (including crack shape correction factor and the crack tip plastic zone correction factor) and $w(x)$ is the weight function. The applicable weight function is determined for example the following way ([2] pp 56-57. - 2.6.5 Weight Functions):

$w(x)=\frac{E}{2 K_{I}} \frac{\partial u_{y}(x)}{\partial a}$,

where $E$ is Young's modulus, $K_{I}$ is a known stress intensity factor, $u_{v}(\mathrm{x})$ is the displacement of the crack face normal to crack plane at a point with $x$-coordinate along the elliptical cracks $a$-axis.

The main disadvantage of this method is, that it is not possible to use the tabulated coefficients from the standard, so it possibly does not include its required conservatism, which means it is might not be directly accepted by the nuclear authorities. However, weight functions can also be generated directly from the $K_{I}$ tables ([2] pp 392-394 9.1.3. Weight Functions for Arbitrary Loading), for example:

$w(x)=$

$=\frac{2}{\sqrt{2 \pi(a-x)}}\left[1+M_{1}\left(1-\frac{x}{a}\right)^{\frac{1}{2}}+M_{2}\left(1-\frac{x}{a}\right)+M_{3}\left(1-\frac{x}{a}\right)^{\frac{3}{2}}\right]$.

The $M_{1}, M_{2}$ and $M_{3}$ coefficients are calculated from the $G_{0}, G_{1}, G_{2}, G_{3}$ values, which are the same coefficients as in eq.2. ( $Q$ is a constant similar to $R_{w}$ )

$M_{1}=\frac{2 \pi}{\sqrt{2 Q}}\left(-G_{0}+3 G_{1}\right)-\frac{24}{5}$

$M_{2}=3$

$M_{3}=\frac{6 \pi}{\sqrt{2 Q}}\left(G_{0}-2 G_{1}\right)-\frac{8}{5}$

Generally speaking, weight functions have the characteristic, they have a singularity at the crack tip:

$w(x) \rightarrow \infty \quad$ if $\quad x \rightarrow a$.

This means using the weight function method leads to improper integral evaluation, which requires higher level numerical calculations. Further than this, if weight function is based on tabulated constants, the coefficients have to be recalculated for all different crack parameters.

\subsection{General weight function}

There is a weight function, applied several times for NPPs to analyse cracks on the inner surface of cylindrical pressure vessels, which is independent from the crack parameters [3]:

$w(x)=\frac{\cos \left(\frac{\pi x}{2 s}\right)}{\sqrt{\sin ^{2}\left(\frac{\pi a}{2 s}\right)-\sin ^{2}\left(\frac{\pi x}{2 s}\right)}}$,

where $s$ is wall thickness.

\section{Polynomial regression using weight function}

In the following a newly developed LSM (LeastSquares Method) based method is presented, which can be used to preserve the conservatisms of the tabulated coefficients declared in the standard, but also resolve their limitations on polynomial regression of the stress function. 


\subsection{Least-Squares Method (LSM) in polynomial regression}

The basis of the method is that the $A_{i}$ parameters of the curve fitting in Equation (1) are determined by the integral formula using the LSM. Universally least squares regression might be presented in the following way:

»The stress function $\sigma^{V E M}(x)$, is usually specified by data points:

$y(i=0, \ldots, n)$

however, using the finite element method the stress function might be a real space function (might include jump discontinuities). The LSM is applicable for both two cases, if stress function is represented by data points or by a real space function. " Assuming linear space, the regression curve is written in the following form:

$\sigma^{A}(x, \underline{A})=\sum_{j=0}^{m} A_{j} \alpha_{j}(x)$,

where $\alpha_{j}(x)$ are base functions, for example:

$\alpha_{j}(x)=\left(\frac{x}{a}\right)^{j}$

and $A_{j}$ is the matching parameter to be calculated, and the $A_{j}$ parameters are collected in a parameter vector: $\underline{A}$.

» The residual function of the approximation:

$r(x)=\sigma^{V E M}(x)-\sigma^{A}(x, \underline{A})$

" According to the specific LSM method, a norm is specified for a function over the crack. The applied norm for data points is usually the root sum of squares:

$\|g\|=\sqrt{\sum_{i=0}^{n} g^{2}\left(x_{i}\right)}$

for functions the integral norm might be used:

$\|g\|=\sqrt{\int_{x=0}^{a} g^{2}(x) d x}$

»The best regression in least squares meaning is the A parameter vector, where the norm of the residual is minimal:

$\|r(x)\| \rightarrow \min$

» The scalar product for data point is usually specified as the following:

$$
\langle f(x), g(x)\rangle=\sum_{i=0}^{n} f\left(x_{i}\right) g\left(x_{i}\right)
$$

for functions it is written in integral form:

$$
\langle f(x), g(x)\rangle=\int_{x=0}^{a} f(x) g(x) d x
$$

"Calculating the $A_{j}$ parameters by solving the following equation system, leads the residual is minimized:

$\underline{\underline{M}} \underline{A}=\underline{B}$

where $\underline{\underline{M}}$ is the stiffness matrix of the base functions:

$M_{i j}=\left\langle\alpha_{i}, \alpha_{j}\right\rangle, i=1, \ldots, m, j=1, \ldots, m$

$\underline{B}$ might be interpreted as the projection of the stress function on the base functions:

$$
B_{j}=\left\langle\alpha_{j}, \sigma^{V E M}\right\rangle, j=1, \ldots, m
$$

\subsection{Weight functions in polynomial regression}

There is no effect on LSM's basic characteristics if integrands in the above integrals multiplied by a properly selected weightfunction (WLSM-Weighted Lest Squares Method). In this case (considering $w(x)$ as the weight function) the integral norm is written in the following form:

$$
\|f\|_{w}=\sqrt{\int_{x=0}^{a} w(x) f^{2}(x) d x}
$$

and scalar product:

$$
\langle f(x), g(x)\rangle_{w}=\int_{x=0}^{a} w(x) f(x) g(x) d x
$$

Obviously, weight functions have large effect on calculation results as it is showed in the example calculations. If the fracture mechanical weight function (according to (3)) is used at the WLSM method as a weight function, we get the best regression for the stress intensity factor in least squares meaning.

Using WLSM for the zero-degree (constant) regression means (using eq. (21) and (22)):

$\alpha_{0}(x)=1 ; \quad \sigma^{A}(x)=A_{0} \cdot 1$

$M_{00}=\int_{x=0}^{a} w(x) \cdot 1 \cdot 1 \cdot d x ; \quad B_{0}=\int_{x=0}^{a} w(x) \cdot 1 \cdot \sigma(x) d x$

So eq. (20) leads to the following:

$M_{00} \cdot A_{0}=B_{0}$

This means $A_{0}$ is calculated as the following:

$$
A_{0}=\frac{\int_{x=0}^{a} w(x) \sigma(x) d x}{\int_{x=0}^{a} w(x) d x}=\frac{K_{I}^{w} / R_{w}}{\int_{x=0}^{a} w(x) d x}
$$


The integral of the weight function over the crack depth is considered constant in this case, since it contains only crack configuration dependent parameters:

$$
\begin{aligned}
& W_{a}=\int_{x=0}^{a} w(x) d x \\
& A_{0}=\frac{K_{I}^{w}}{R_{w} \cdot W_{a}}
\end{aligned}
$$

Considering:

$K_{I}^{G}=A_{0} R_{0} G_{0}$

leads:

$$
K_{I}^{G}=\frac{K_{I}^{w}}{R_{w} \cdot W_{a}} R_{0} G_{0}=\frac{R_{0}}{R_{w}} \frac{G_{0}}{W_{a}} K_{I}^{w}
$$

This means, the two calculation results will be characteristically identical, the difference between them are in the multiplicative constants (including any other conservatisms in the case of the nuclear code). Which means using the above regression, leads to (proportionally) the same errors as using the weight function method for the same stress field. Using the conventional LSM approach, we might get characteristically different results, like it is shown at the calculation examples. Considered from the above equations, the constant of the weight function method does not affect the stress-curve regression, so the following assumption might be used:

$$
R_{w}=1
$$

\subsection{Test calculations}

The PTSLab computer code - developed by the author - was used for calculating the following examples, which show the practical use of the above described methods. PTSLab earlier was used by VEIKI E+ Ltd. to perform parametric calculations supporting the development of the PTS (No. 3.18) regulatory guide and also for checking the results of the PTS calculations submitted to the Hungarian Atomic Energy Authority by Paks NPP as a part of the licence renewal application of the WWER440 units [4].

Test calculations are based on the following main parameters and usual crack configurations of WWER 440/213 units:

" Main dimensions of the pressurized vessel: inner diameter: $d_{0}=3542 \mathrm{~mm}$; cladding thickness: $t_{c l}=9 \mathrm{~mm}$;

base material thickness: $t_{b}=140 \mathrm{~mm}\left(t=t_{c l}\right.$ $\left.+t_{b}\right)$

" Crack characteristics:

axial, semi-elliptical surface cracks, depth: $\mathrm{a}=16,23$ and $30 \mathrm{~mm}$; crack shape factor: a $/ \mathrm{c}=1 / 3$.

Test cases are set to:

" highlight the typical errors of the basic polynomial regressions;

" include typical stress fields from the practical PTS calculations:

- stress discontinuity caused by the different heat-expansion coefficient of cladding and base metal;

- residual stress in welds from the regulations;

- critical case from a real-world calculation;

" parameters of the stress distribution polynomials are a set, so that calculations with the Westergaard method (number 6.) using a crack with a depth of $a=23 \mathrm{~mm}$ would give the same results on the stress intensity factor for most cases;

According to the conditions above the following stress distribution functions are used:
a) Constant stress distribution: $\sigma^{F E M}(x)=55.3 \mathrm{MPa}$
b) 1st order stress distribution: $\sigma^{\mathrm{FEM}}(x)=92.9-383.77 \cdot(x / t)$ $\mathrm{MPa}$
c) 2ndorderstress distribution: $\sigma^{\mathrm{FEM}}(x)=92.9-3098.49 \cdot(x / t) 2$ $\mathrm{MPa}$
d) 3rd order stress distribution: $\sigma^{F E M}(x)=92.9-7806.33 \cdot(x)$ t) $2+34888.75 \cdot(x / t) 3$

e) Constant stress distribution with discontinuity at the interface of cladding (modelling the different heatexpansion coefficient of cladding and base metal):

$$
\sigma^{F E M}(x)=\left\{\begin{array}{c}
224,4, \text { if } x / t<0,06 \\
-3,57 \text { otherwise }
\end{array}\right.
$$

f) Residual stress (according to Hungarian regulations):

$$
\sigma^{F E M}(x)=\left\{\begin{array}{c}
60, \text { if } x / t<0,06 \\
60 \cos \left(2 \pi \frac{x-t_{c l}}{t_{b}}\right) \text { otherwise }
\end{array}\right.
$$

g) Stress distribution from the PTS analysis, during an accident (LBLOCA);

\section{Results and Discussion}

The calculation results are shown in the following figures. Based on the results, these conclusions are made:

"Methods rely on fixed base point regressions correlate well with the other results as long as the polynomial degree of the stress distribution function is not greater than the degree of the method (for linear approximation, these are the cases titled $a$., b.). Deviations with larger crack sizes are greater. The difference is 
Table 1: List of applied methods

\begin{tabular}{|c|c|c|c|}
\hline Id & Short name & Main characteristics & Notes \\
\hline \multicolumn{4}{|c|}{ Methods based on regression } \\
\hline 1. & ASME linear & $\begin{array}{l}\text { 1st order linear regression on fixed base } \\
\text { points, using tabular coefficients }\end{array}$ & Source: [1] Appendix A-3000 \\
\hline 2. & $\begin{array}{l}\text { ASME } \\
\text { polynomial }\end{array}$ & $\begin{array}{l}\text { 3rd order regression on fixed base } \\
\text { points, using tabular coefficients }\end{array}$ & Source: [1] Appendix A-3000 \\
\hline 3. & CEA & $\begin{array}{l}\text { 1st and 3rd order combined regression } \\
\text { on fixed base points, using tabular } \\
\text { coefficients }\end{array}$ & $\begin{array}{l}\text { Method is developed by the French Alternative Energies } \\
\text { and Atomic Energy Commission (CEA) [5]. Based on FEM } \\
\text { calculations, taking account the discontinuity of the stress } \\
\text { function at the cladding. }\end{array}$ \\
\hline 4. & ASME LSM & $\begin{array}{l}\text { 3rd order regression based on LSM, } \\
\text { using tabular coefficients }\end{array}$ & $\begin{array}{l}\text { Method is described above in subsection 2.1. Source of } \\
\text { tabular data: [1] Appendix A-3000 }\end{array}$ \\
\hline \multicolumn{4}{|c|}{ Weight function methods } \\
\hline 5. & ASMEWF & $\begin{array}{l}\text { Weight functions calculated from 1st } \\
\text { order regressions tabular coefficients. }\end{array}$ & $\begin{array}{l}\text { The weight function from eq (5) is used. (Source: [2] pp } \\
\text { 392-394) }\end{array}$ \\
\hline 6. & Westergaard & $\begin{array}{l}\text { General weight function for semi- } \\
\text { elliptical surface cracks. }\end{array}$ & $\begin{array}{l}\text { Source: [3]. The method is using the weight function } \\
\text { shown above in eq. (10). }\end{array}$ \\
\hline \multicolumn{4}{|c|}{ Regression using weight function } \\
\hline 7. & $\begin{array}{l}\text { ASMEWLSM } \\
\text { tabular }\end{array}$ & $\begin{array}{l}\text { Polynomial regression using weight } \\
\text { functions calculated from the } 1 \text { st order } \\
\text { regressions tabular coefficients. }\end{array}$ & $\begin{array}{l}\text { Method is described above in subsection 2.1. The weight } \\
\text { function from eq (5) is used for the integration. }\end{array}$ \\
\hline 8. & $\begin{array}{l}\text { ASME WLSM } \\
\text { Westergaard }\end{array}$ & $\begin{array}{l}\text { Polynomial regression using } \\
\text { Westergaard weight functions }\end{array}$ & $\begin{array}{l}\text { Method is described above in subsection 2.1. The weight } \\
\text { function from eq (10) is used for the integration. }\end{array}$ \\
\hline
\end{tabular}

somewhat reduced when using the LSM method (without weight function).

"An exception to the above is the CEA method, which in all cases contains less conservatism than the others, besides this the difference compared to the weight function methods does not increase even if there is a discontinuity of the stress distribution function at the cladding interface (case e.). However, the difference is more significant for the residual stress (case f.), where the approximation is no longer capable to accommodate to the stress distribution function. Concluding the above, the CEA method is quite well applicable in general, but its conservatism does not reach the level of ASME's.

"Methods based on the weight functions give very similar results in a consistent manner. For methods based on the ASME approximation, this consistency is particularly significant (methods 7., 8.), regardless of the used weight function.

»PTS transient:

- Methods rely on fixed point regression has a different character in the stress intensity values over time, compared to the other results. The difference is the most significant for the ASME method using linear approximation. Although the results using the (EA method is similar in characteristics as the results with the weight function methods, it includes much less conservatism.

- The computationally intensive methods give very close results to each-other (5., 6., 7., 8.).

\section{Conclusions}

Based on the above presented characteristics and calculation results of the developed method, it can be concluded the method corresponds to the approximation methods of the nuclear code, generally accepted by the authorities, as the interpolation coefficients are calculated and also the approximation itself might be presented graphically. In addition, it has a consistent, similar characteristic to the general weight function based methods, which means it produces acceptable results, even for complex stress distributions. Although one feature of the method is that a well-implemented numerical calculation is needed which means higher computational requirements, but these are significantly lower than the computational requirements of analyses of a directly modelled cracks using FEM. 
Semi-elliptical surface crack

depth: $\mathrm{a}=16 \mathrm{~mm}$; crack shape factor: $\mathrm{a} / \mathrm{c}=1 / 3$

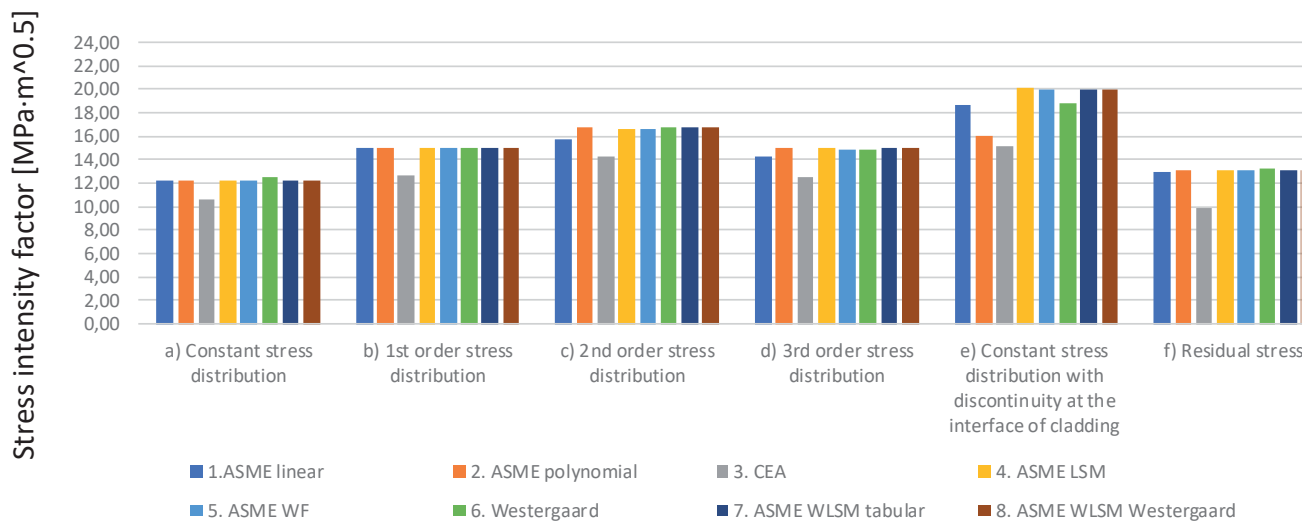

Semi-elliptical surface crack

depth: $\mathrm{a}=23 \mathrm{~mm}$; crack shape factor: $\mathrm{a} / \mathrm{c}=1 / 3$

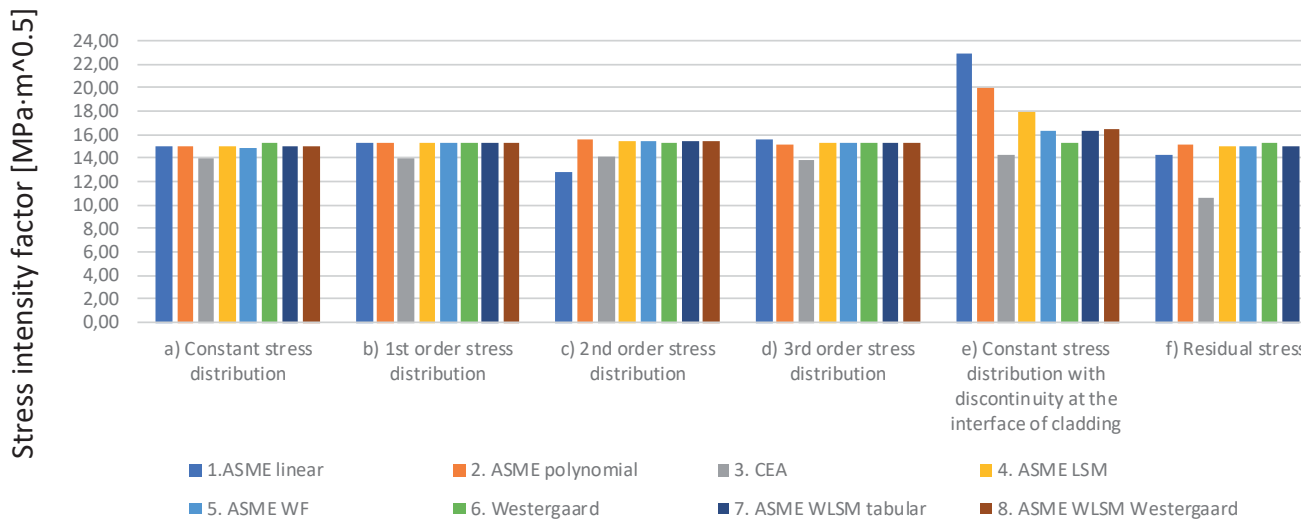

Semi-elliptical surface crack

depth: $\mathrm{a}=30 \mathrm{~mm}$; crack shape factor: $\mathrm{a} / \mathrm{c}=1 / 3$

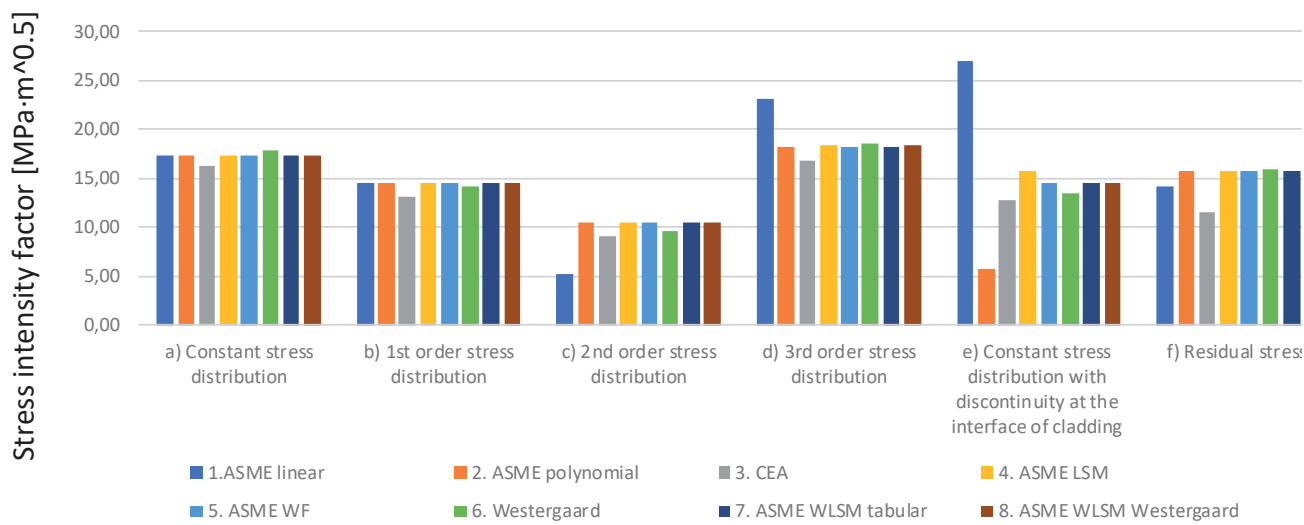

Figure 1: Stress intensity calculation results for single stress distribution test (cases a-f 
PTS LBLOCA transient

Semi-elliptical surface crack, depth: $a=30 \mathrm{~mm}$; crack shape factor: $\mathrm{a} / \mathrm{c}=1 / 3$

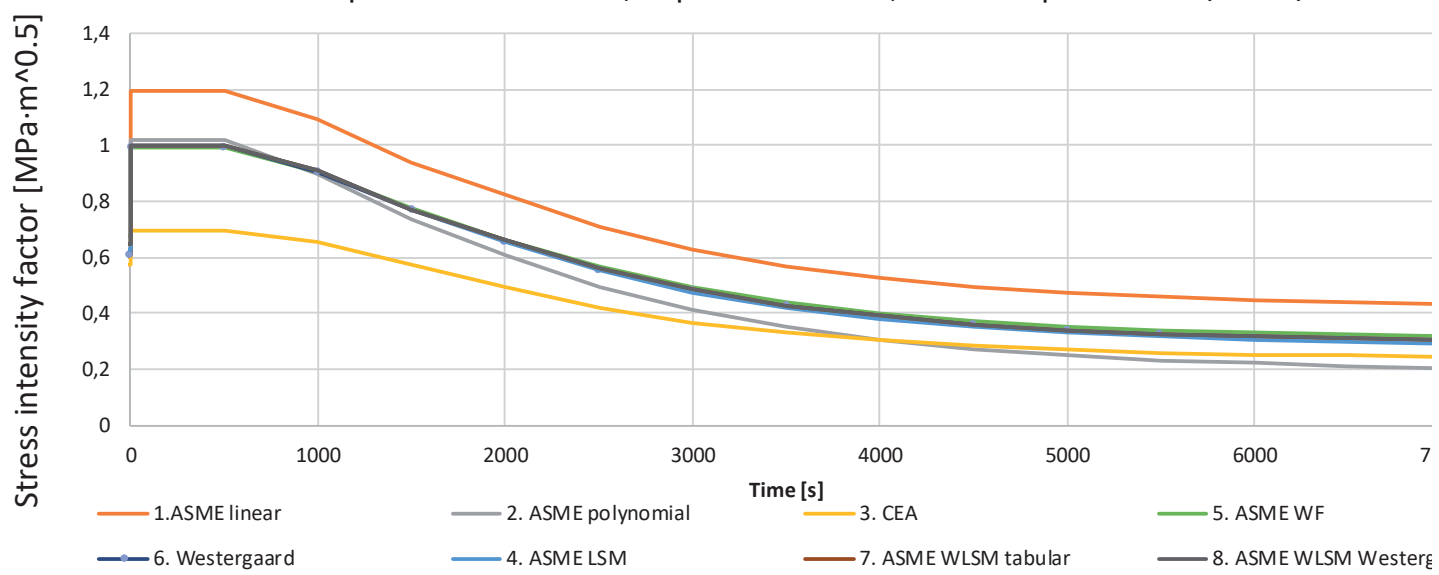

Figure 2: Stress intensity results for the LBLOCA test calculations (case g)

\section{References and Notes}

1. ASME Boiler \& Pressure Vessel Code (2007). Division 1 Section XI, Rules for Inservice Inspection of Nuclear Power Plant Components. ASME, The American Society of Mechanical Engineers. New York.

2. Anderson, T. L. (2011). Fracture Mechanics - Fundamentals and Applications. CRC Press Taylor \& Francis, Boca Raton.

3. Schmitt W., Keim E. (1979). Linear Elastic Analysis of Semi-Elliptical Axial Surface Cracks in a Hollow Cylinder. International Journal of Pressure Vessels and Piping, Volume 7, Issue 2, pp 105-118.

4. Pálfi T., Osztheimer M, Janosiné Bíró Á. (2011) PTS vizsgálatok részletes ellenőrző számításainak elvégzése. Scientific report for the Hungarian Atomic Energy Authority. VEIKI E+ Ltd.., Budapest, Hungary.

5. Marie, S.; Menager, Y.; Chapuliot, S. (2005). Stress Intensity Factors for Underclad and Through Clad Defects in a Reactor Pressure Vessel Submitted to a Pressurised Thermal Shock. International Journal of Pressure Vessels and Piping, Volume 82, Issue 10, pp 746-760. 